\title{
Neurologic outcome of postanoxic refractory status epilepticus after aggressive treatment
}

Simone Beretta, MD, PhD, Anna Coppo, MD, Elisa Bianchi, MSc, Clara Zanchi, MD, Davide Carone, MD, Andrea Stabile, MD, Giada Padovano, MD, Endrit Sulmina, MD, Alice Grassi, MD, Graziella Bogliun, MD, Giuseppe Foti, MD, Carlo Ferrarese, MD, PhD, Antonio Pesenti, MD, Ettore Beghi, MD, and Leonello Avalli, MD Neurology ${ }^{\circledR}$ 2018;00:1-10. doi:10.1212/WNL.0000000000006615

\begin{abstract}
\section{Objective}

To investigate neurologic outcome of patients with cardiac arrest with refractory status epilepticus (RSE) treated with a standardized aggressive protocol with antiepileptic drugs and anesthetics compared to patients with other EEG patterns.
\end{abstract}

\section{Methods}

In the prospective cohort study, 166 consecutive patients with cardiac arrest in coma were stratified according to 4 independent EEG patterns (benign, RSE, generalized periodic discharges [GPDs], malignant nonepileptiform) and multimodal prognostic indicators. Primary outcomes were survival and cerebral performance category (CPC) at 6 months.

\section{Results}

RSE occurred in 36 patients (21.7\%) and was treated with an aggressive standardized protocol as long as multimodal prognostic indicators were not unfavorable. RSE started after $3 \pm 2.3$ days after cardiac arrest and lasted $4.7 \pm 4.3$ days. A benign EEG pattern was recorded in 76 patients $(45.8 \%)$; a periodic pattern (GPDs) was seen in 13 patients (7.8\%); and a malignant nonepileptiform EEG pattern was recorded in 41 patients (24.7\%). The 4 EEG patterns were highly associated with different prognostic indicators (low-flow time, clinical motor seizures, N20 responses, neuron-specific enolase, neuroimaging). Survival and good neurologic outcome (CPC 1 or 2 ) at 6 months were $72.4 \%$ and $71.1 \%$ for benign EEG pattern, $54.3 \%$ and $44.4 \%$ for RSE, $15.4 \%$ and $0 \%$ for GPDs, and $2.4 \%$ and $0 \%$ for malignant nonepileptiform EEG pattern, respectively.

\section{Conclusions}

Aggressive and prolonged treatment of RSE may be justified in patients with cardiac arrest with favorable multimodal prognostic indicators.

\section{Correspondence}

Dr. Beretta

simone.beretta@unimib.it

From the Epilepsy Center (S.B., C.Z., D.C. A.S., G.P., G.B., C.F.), Department of Neurology, and Department of Intensive Care (A.C., E.S., A.G., G.F., L.A.), San Gerardo Hospital ASST Monza, University of Milano Bicocca, Monza; Department of Neuroscience (E.B., E.B.), IRCCS Mario Negri Institute for Pharmacological Research; and Department of Anesthesia (A.P.), Critical Care and Emergency, IRCCS Ospedale Maggiore Policlinico, Milano, Italy.

Go to Neurology.org/N for full disclosures. Funding information and disclosures deemed relevant by the authors, if any, are provided at the end of the article. 


\section{Glossary}

ACNS = American Clinical Neurophysiology Society; AED = antiepileptic drug; $\mathbf{c E E G}=$ continuous EEG; $\mathbf{C I}=$ confidence interval; CPC = cerebral performance category; GPD = generalized periodic discharge; ICU = intensive care unit; NCSE = nonconvulsive status epilepticus; NSE = neuron-specific enolase; $\mathrm{OR}=$ odds ratio; RSE = refractory status epilepticus; TELSTAR = Treatment of Electroencephalographic Status Epilepticus After Cardiopulmonary Resuscitation.

Prognostication of neurologic outcome in patients in coma after cardiac arrest requires a multimodal diagnostic approach to assess the severity of postanoxic encephalopathy, which includes clinical examination, EEG pattern, somatosensory evoked potentials, neuron-specific enolase (NSE), and neuroimaging. ${ }^{1}$

Refractory status epilepticus (RSE) has been reported in up to $30 \%$ of comatose patients after cardiac arrest with continuous EEG (cEEG), ${ }^{2-4}$ is frequently associated with clinical motor seizures (myoclonic, clonic, or tonic-clonic), ${ }^{5}$ and is typically resistant to moderate-intensity, unstandardized treatment. ${ }^{6}$

The standardized American Clinical Neurophysiology Society (ACNS) terminology of critical care EEG ${ }^{7}$ and the Salzburg criteria for nonconvulsive status epilepticus (NCSE) ${ }^{8,9}$ allow a clear distinction of 2 EEG patterns, which are frequently reported under the same term "status epilepticus." Sharply contoured generalized periodic discharges (GPDs; GPDs+, previously called generalized periodic epileptic discharges) have consistently been reported as a highly malignant pattern associated with a poor neurologic prognosis. ${ }^{10} \mathrm{RSE}$ without periodic discharges has also been associated with poor prognosis, ${ }^{11}$ but a subset of these patients might achieve a good functional recovery. ${ }^{12,13}$

Both the intensity and duration of treatment of RSE in postanoxic patients are controversial and raise ethical issues of therapeutic obstinacy vs premature withdrawal of life support. $^{14,15}$

In the present study, we investigated survival and long-term neurologic outcome in consecutive postanoxic patients with RSE without periodic discharges treated with an aggressive standardized protocol compared to patients with a benign EEG pattern, a nonepileptiform malignant EEG pattern, and GPDs.

\section{Methods}

\section{Study protocol approvals, registrations, and patient consents}

This prospective cohort study explored survival and long-term neurologic outcome of postanoxic patients with RSE after aggressive standardized treatment. This study was approved by the Ethics Committee of San Gerardo Hospital, Monza, Italy. Consecutive patients in coma after cardiac arrest admitted between January 2011 and May 2016 in the hospital's cardiac intensive care unit (ICU) were included in the study.
To be included in the cohort, patients had to be in a coma for $>24$ hours after cardiac arrest. Patients who regained consciousness or died $<24$ hours after cardiac arrest were excluded.

Data collection was performed by review of medical records for demographics and clinical data, including clinical motor seizures, brainstem reflexes, NSE, somatosensory evoked potentials, and neuroimaging. EEG patterns within the first 5 days from cardiac arrest were independently reviewed and categorized by 3 neurophysiologists (see below). Neurologic outcome was assessed at 6 months after cardiac arrest by telephone contact (see below).

\section{Aggressive standardized treatment of postanoxic status epilepticus}

Patients were treated according to the local institutional protocol for reducing brain damage after cardiac arrest (figure 1). This includes targeted temperature management at $34^{\circ} \mathrm{C}$ for 24 hours, simplified 4-channel cEEG monitoring applied within 24 hours from cardiac arrest, and on-call neurologic consultations with an epilepsy specialist. Standard 18-channel EEG recording was performed twice in the first 5 days or more frequently if clinically indicated, in addition to cEEG.

A multimodal prognostic approach was applied in all cases; i.e., an unfavorable profile was considered when most indicators converged indicating a poor prognosis, while a not unfavorable (i.e., potentially favorable) profile was considered when most indicators converged against a poor prognosis. Brainstem reflexes (pupillary, corneal) were performed within 24 hours and if bilaterally absent were considered indicators of poor prognosis. NSE levels were performed at 48 hours, and we adopted the cutoff of $>68 \mathrm{ng} / \mathrm{mL}$ as an indicator of poor prognosis. ${ }^{16}$ Median nerve somatosensory evoked potentials were performed at 72 hours if patients remained in coma, and if bilaterally absent, they were considered indicators of poor prognosis. Neuroimaging (CT or MRI) was performed if clinically indicated and feasible. If moderate to severe anoxic brain injury was present (see below), it was considered an indicator of poor prognosis.

Early identification of prognostic EEG patterns (see below) was performed by on-call epilepsy specialists within the first 5 days after cardiac arrest, with multiple consultations as needed. If status epilepticus was detected by cEEG during hypothermia or rewarming, IV benzodiazepines and IV valproate, levetiracetam, and/or phenytoin were administered, followed within 60 minutes by a first anesthetic-induced coma with propofol and/ or midazolam for 48 hours if epileptic activity was refractory to 
Figure 1 Standardized protocol for treatment of hypoxic-ischemic encephalopathy in the intensive care unit

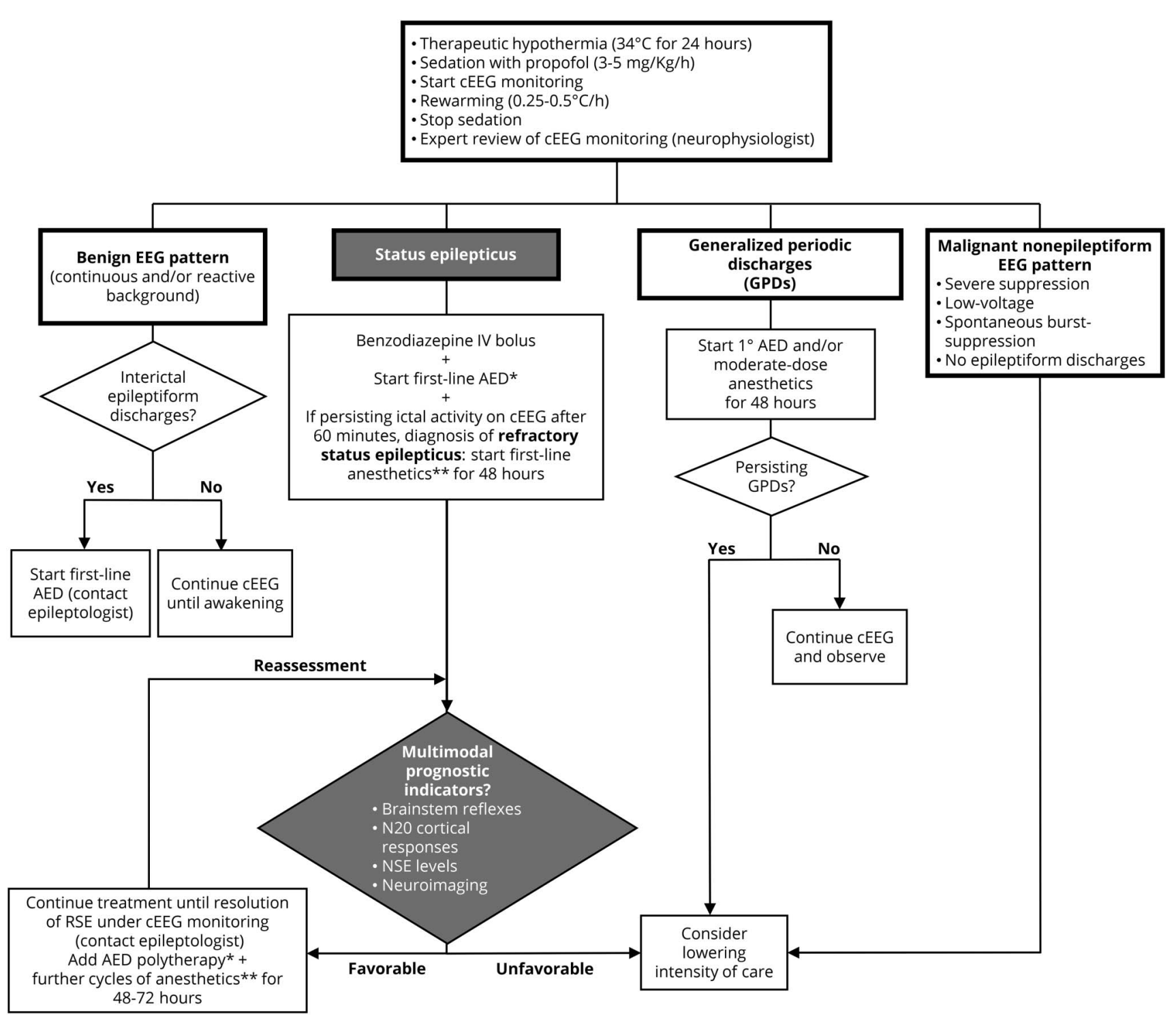

$\mathrm{AED}=$ antiepileptic drug; BSR = burst suppression rate (percent of time the waveform is isoelectric over the previous 60 seconds); $\mathrm{CEEG}=\mathrm{continuous} \mathrm{EEG;} \mathrm{NSE}$ = neuron-specific enolase; RSE = refractory status epilepticus. *Antiepileptic drugs. First-line: valproate $30 \mathrm{mg} / \mathrm{kg}$ IV bolus + $1.5 \mathrm{mg} / \mathrm{kg}$ IV maintenance; levetiracetam $40 \mathrm{mg} / \mathrm{kg}$ IV bolus + 2 to $5 \mathrm{mg} / \mathrm{kg}$ IV maintenance; phenytoin (if contraindication to valproate/levetiracetam and absent cardiac risks) $20 \mathrm{mg} / \mathrm{kg}$ IV bolus $+5 \mathrm{mg} / \mathrm{kg}$ oral maintenance (frequent plasma levels to adjust dose). Second-line: lacosamide $400 \mathrm{mg}$ IV bolus $+400 \mathrm{mg} / \mathrm{d}$ IV maintenance; topiramate

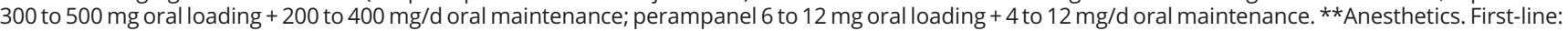
propofol 1 to $2 \mathrm{mg} / \mathrm{kg}$ IV bolus +2 to $6 \mathrm{mg} / \mathrm{kg} / \mathrm{h}$ IV maintenance, target burst suppression guided by cEEG (60\%-70\% BSR) \pm midazolam. Second-line: thiopental $5-15 \mathrm{mg} / \mathrm{kg}$ IV bolus + 0.5 to $10 \mathrm{mg} / \mathrm{kg} / \mathrm{h}$ IV maintenance, target burst suppression guided by cEEG (60\%-70\% BSR); ketamine 1.5 to 3 mg/kg IV bolus + $1-5 \mathrm{mg} /$ kg/h IV maintenance, target typical ketamine pattern, no epileptiform discharges \pm midazolam.

treatment, guided by cEEG monitoring. Other antiepileptic drugs (AEDs; lacosamide, topiramate, perampanel) and/or cycles of anesthetics-induced coma (propofol, midazolam, thiopental, ketamine) were administered if needed, guided by cEEG and available prognostic indicators (figure 1 provides details). Our protocol for aggressive standardized treatment of postanoxic status epilepticus was developed from the results of previous expert opinions on this topic. . $^{17,18}$

\section{Study definitions}

Status epilepticus was defined according to the International League Against Epilepsy classification. ${ }^{19}$ In accordance with the Salzburg EEG criteria, ${ }^{8}$ a frequency of epileptiform discharges ( diagnosis of NCSE. A minimum duration of 30 minutes of electrographic seizure activity was used for the diagnosis of an episode of NCSE. No patients included in this study had previous epileptic encephalopathy.

Status epilepticus was defined as refractory (RSE) after failure of benzodiazepines and a first IV AED and superrefractory after failure of a first cycle ( $\geq 48$ hours) of anesthetics-induced burst-suppression pattern (burst suppression rate $>70 \%)$. 
Motor seizures were defined as any observable myoclonic, clonic, or tonic-clonic manifestation occurring during the entire stay in the ICU.

Status myoclonus was defined as continuous and generalized myoclonic jerks with a duration of $>30$ minutes ${ }^{1}$ within the first 5 days after cardiac arrest.

GPDs were defined as sharply contoured, sharp or spiky discharges showing a periodic pattern ${ }^{7}$ with a frequency $<2.5$ Hz. A minimum duration of 30 minutes of continuous periodic discharges was used for the diagnosis of an episode of GPDs. Periodic discharges with a frequency $>2.5 \mathrm{~Hz}$ were considered status epilepticus.

EEG background activity was categorized as continuous $(<10 \%$ periods of attenuation or suppression) or discontinuous ( $>10 \%$ periods of attenuation or suppression, including spontaneous burst suppression and suppression).

EEG background reactivity was defined as any response elicited by auditory or noxious stimuli, excluding stimulusinduced rhythmic periodic or ictal discharges.

MRI evidence of anoxic brain injury was dichotomized as mild or moderate to severe according to diffusion-weighted imaging or fluid-attenuated inversion recovery abnormalities in the cortical gray matter and deep basal ganglia. ${ }^{20}$
CT evidence of anoxic brain injury was dichotomized as mild or moderate to severe according to the ratio of gray matter to white matter and diffuse cerebral edema. ${ }^{21}$

\section{Prognostic EEG pattern}

Four mutually exclusive EEG patterns within the first 5 days after cardiac arrest were defined as follows: (1) benign EEG pattern: continuous or reactive (or both) EEG background activity at any time point, with no episodes of status epilepticus or GPDs; (2) RSE pattern: $\geq 1$ episodes of RSE at any time point, with no episodes of GPDs $<2.5 \mathrm{~Hz}$ at any time, independently of EEG background activity; (3) GPD pattern: $\geq 1$ episodes of GPDs $<2.5 \mathrm{~Hz}$ at any time point, independently of EEG background activity or RSE; and (4) malignant nonepileptiform EEG pattern: consistently discontinuous and unreactive EEG background activity, with no episodes of status epilepticus or GPDs at any time.

Examples of the 4 prognostic EEG patterns are illustrated in figure 2. Three neurophysiologists independently reviewed the EEGs of different patients and categorized them in the 4 EEG patterns. The interrater agreement was tested with the Fleiss $\kappa$ on 25 randomly selected EEG recordings from the same patients ( $15 \%$ of the study population).

\section{Sample size}

Assuming that mortality in patients meeting the inclusion criteria and without RSE is 50\%, with 206 patients enrolled, the study had sufficient statistical power $(80 \%)$ to detect

Figure 2 Prognostic EEG patterns in post-cardiac arrest patients
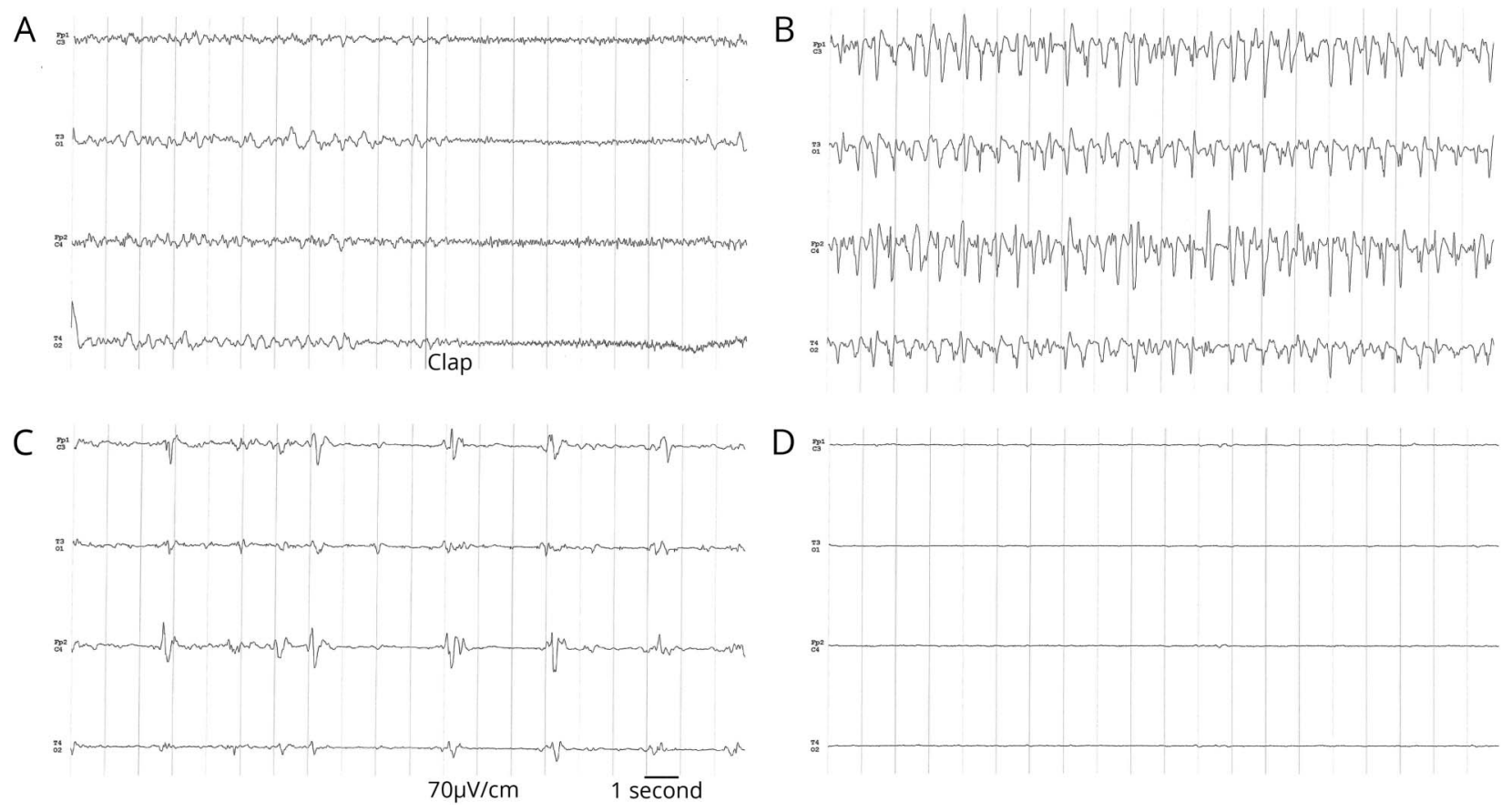

Representative epochs from 4-channel continuous EEG monitoring of patients with (A) benign EEG pattern, (B) refractory status epilepticus pattern, (C) generalized periodic discharge pattern, and (D) malignant nonepileptiform EEG pattern. 
a 20\% absolute difference between patients with and without RSE with a $5 \%$ level of significance $(p=0.05)$.

\section{Study outcomes}

Primary outcomes were survival and neurologic disability, assessed with the cerebral performance category (CPC), at 6 months after cardiac arrest. ${ }^{22}$ All patients or caregivers were contacted by phone call; no patient was lost to follow-up. $\mathrm{CPC}$ ranges from 1 to 5 , with 1 representing intact function and 5 representing brain death. CPC was dichotomized as follows: patients with a CPC of 1 or 2 were classified as having good neurologic outcome, and patients with a CPC of $\geq 3$ were classified as having poor neurologic outcome.

\section{Statistical analysis}

Descriptive statistics were performed in the study population for the main demographic and clinical variables. Data are reported as frequencies and percentages for categorical variables or as medians and interquartile ranges for continuous variables. Selected prognostic indicators (clinical motor seizures, low-flow time, N20, NSE, neuroimaging) were described separately for each EEG pattern and compared by use of the $\chi^{2}$ or the Wilcoxon-Mann-Whitney test. Clinical characteristics of RSE (onset, duration, response to treatment) were described with means, SDs, ranges, frequencies, percentages, and cumulative incidence functions. The effect of demographic and clinical variables on study outcomes was evaluated with univariable logistic regression models. Results are reported as odds ratios (ORs) with 95\% confidence intervals (95\% CIs). The significance level was set at 0.05 . Statistical analyses were performed with the SAS statistical package (version 9.4; SAS Institute, Cary, NC).

\section{Data availability}

Anonymized data will be shared on request from any qualified investigator.

\section{Results}

\section{Study population}

The initial cohort consisted of 206 consecutive patients with cardiac arrest; 40 patients were excluded because they died within the first 24 hours and were not EEG monitored. All remaining 166 patients survived the first 24 hours, had cEEG monitoring, and were included in the analysis.

Therapeutic hypothermia $\left(34^{\circ} \mathrm{C}\right)$ for 24 hours, followed by gradual rewarming over the next 12 hours, was applied in 148 patients $(89.1 \%)$. Demographic and clinical characteristics of the study population are shown in table 1. Median age was 61 years (interquartile range 51-71 years). Most patients had preserved brainstem reflexes (pupillary $84.1 \%$, corneal $70.1 \%$ ) and bilaterally present cortical N20 responses (82.5\%). Early brain imaging with MRI or CT (within 7 days) was performed in 87 patients (52.4\%) and showed moderate to severe anoxic brain injury in 33 patients (37.9\%).

\section{Prognostic EEG patterns}

cEEG monitoring was used to categorize patients in the 4 distinct, mutually exclusive EEG patterns within the first 5 days of recording: 76 patients (45.8\%) had a benign EEG pattern; 36 patients $(21.7 \%)$ had RSE; 13 patients (7.8\%) had GPDs; and 41 patients $(24.7 \%)$ had a malignant nonepileptiform EEG pattern (table 2$)$. Interrater agreement was very high $(\kappa=0.92)$.

Table 1 Clinical characteristics of the study population

\begin{tabular}{|c|c|c|c|}
\hline & Unit & Median or $\mathbf{n}(\%)$ & IQR \\
\hline Age & Years & 61.0 & $51.0-71.0$ \\
\hline Days in ICU & Days & 7.5 & $4.0-15.0$ \\
\hline Sex & Male, n (\%)/female, n (\%) & $120(72.7) / 46$ (27.7) & \\
\hline No-flow time & Minutes & 1.0 & $1.0-7.5$ \\
\hline Low-flow time & Minutes & 28.0 & $14.5-53.0$ \\
\hline Out-of-hospital cardiac arrest & n (\%) & $112(67.5)$ & \\
\hline ECMO & n (\%) & $84(50.6)$ & \\
\hline Hypothermia & n (\%) & $148(89.1)$ & \\
\hline Pupillary reflex ${ }^{a}$ & Bilaterally present, n (\%) & $127(84.1)$ & \\
\hline Corneal reflex ${ }^{a}$ & Bilaterally present, n (\%) & $103(70.19)$ & \\
\hline NSE & $\mu g / L$ & 48.0 & $31.6-98.7$ \\
\hline Cortical N20 response ${ }^{b}$ & Bilaterally absent, n (\%) & $20(17.5)$ & \\
\hline MRI or CT (within 7 d) & Performed in n (\%) & $87(52.4)$ & \\
\hline
\end{tabular}

Abbreviations: ECMO = extracorporeal membrane oxygenation; ICU = intensive care unit; IQR = interquartile range; NSE = neuron-specific enolase. ${ }^{a}$ Missing data in $9.0 \%$ for pupillary reflex and $11.4 \%$ for corneal reflex.

b Missing data in 31.3\% for cortical N20 responses (patients either awakened or died before performing median nerve somatosensory evoked potentials at 72 hours). 
Table 2 EEG patterns, clinical seizures, and selected prognostic indicators

\begin{tabular}{|c|c|c|c|c|c|c|c|}
\hline $\begin{array}{l}\text { EEG pattern (day } \\
0-5 \text { ) }\end{array}$ & n (\%) & $\begin{array}{l}\text { Clinical motor } \\
\text { seizures, } n \text { (\% of } \\
\text { pattern) }\end{array}$ & $\begin{array}{l}\text { Status myoclonus, } \\
\mathrm{n} \text { ( } \% \text { of motor } \\
\text { seizures) }\end{array}$ & $\begin{array}{l}\text { Low-flow } \\
\text { time } \\
\text { (median), } \\
\text { min }\end{array}$ & $\begin{array}{l}\text { NSE } \\
\text { (median), } \mu g / \\
\text { L }\end{array}$ & $\begin{array}{l}\text { Absent }^{\mathrm{a}} \mathrm{N} 20, \\
\mathrm{n}(\% \text { of } \\
\text { pattern) }\end{array}$ & $\begin{array}{l}\text { MRI-CT anoxic } \\
\text { injury, }{ }^{b} \mathrm{n}(\% \text { of } \\
\text { subset) }\end{array}$ \\
\hline $\begin{array}{l}\text { Benign EEG } \\
\text { pattern }\end{array}$ & $\begin{array}{l}76 \\
(45.8)\end{array}$ & $6(7.8)$ & $0(0)$ & 20 & 36 & $3(4.5)$ & $7(19.4)$ \\
\hline RSE pattern & $\begin{array}{l}36 \\
(21.7)\end{array}$ & $16(44.4)$ & $3(18.7)$ & 26 & 47 & $4(16.7)$ & $4(22.2)$ \\
\hline GPD pattern & $\begin{array}{l}13 \\
(7.8)\end{array}$ & $8(61.5)$ & $5(62.5)$ & 40 & 99 & $6(54.6)$ & $5(62.5)$ \\
\hline $\begin{array}{l}\text { Malignant } \\
\text { nonepileptiform } \\
\text { EEG pattern }\end{array}$ & $\begin{array}{l}41 \\
(24.7)\end{array}$ & $4(9.8)$ & $3(75.0)$ & 56 & 248 & $7(58.3)$ & $17(68.5)$ \\
\hline Total & $\begin{array}{l}166 \\
(100)\end{array}$ & $34(20.5)$ & $11(32.3)$ & 28 & 48 & 20 & 33 (37.9) \\
\hline
\end{tabular}

Abbreviation: GPD = generalized periodic discharge; NSE = neuron-specific enolase; RSE = refractory status epilepticus.

${ }^{a}$ Bilaterally absent cortical N20 responses missing data in 31.3\% for cortical N20 responses (patients either awakened or died before performing somatosensory median nerve evoked potentials at 72 hours).

${ }^{\mathrm{b}}$ Moderate to severe degree of anoxic brain injury based on MRI or CT imaging $<7$ days (performed in a subset of 87 patients).

The 4 EEG patterns were highly associated with different prognostic indicators (clinical motor seizures $p<0.0001$, lowflow time $p<0.0001$, N20 $p<0.0001$, NSE $p<0.0001$, neuroimaging $p=0.0003$ ). RSE displayed similarities with the benign EEG pattern, while GPDs displayed similarities with the malignant nonepileptiform EEG pattern for low-flow time $(p<0.0001)$, N20 $(p<0.0001)$, NSE $(p<0.0001)$, and neuroimaging $(p<0.0001)$ but not for clinical motor seizures $(p=0.8673)$, as shown in table 2.

\section{Characteristics and treatment of RSE}

Status epilepticus occurred in 43 (25.9\%) patients and met the criteria for RSE pattern in $36(21.7 \%)$ patients while in 7 (4.2\%) patients evolved from an RSE to GPDs within the first
5 days. These patients were considered to have GPDs, together with 6 other $(3.6 \%)$ patients who exhibited GPDs exclusively.

Clinical motor seizures (myoclonic, clonic, or tonic-clonic) during the ICU stay were more prevalent in patients with GPDs (61.5\%) compared to those with RSE (44.4\%), as shown in table 2. Status myoclonus was the most common type of clinical seizure manifestation in GPDs (62.5\%), while it was infrequent in RSE (18.7\%). All patients with status myoclonus had poor neurologic outcome.

Timing of RSE is shown in figure 3. The mean onset of RSE was $3 \pm 2.3$ days after cardiac arrest. The mean duration of

Figure 3 Time course of RSE in post-cardiac arrest patients
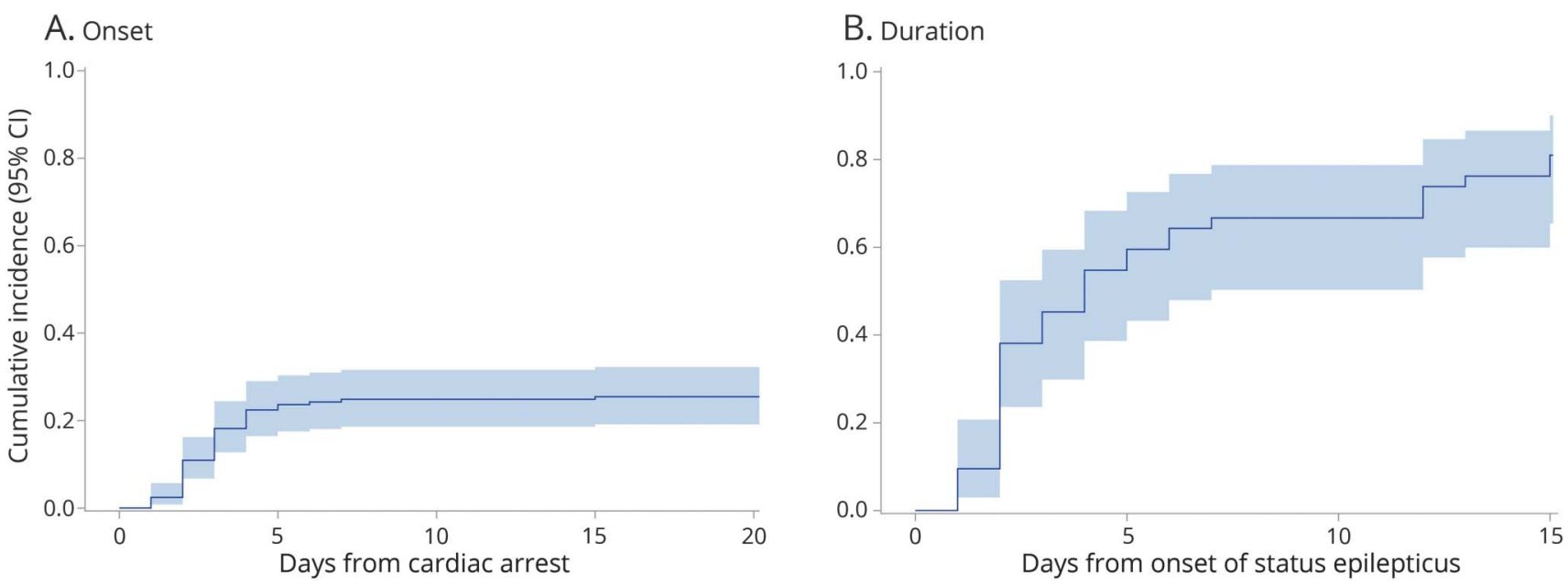

Cumulative incidence with 95\% confidence interval (CI) is shown for (A) onset and (B) duration of refractory status epilepticus (RSE). 
Table 3 Effect of EEG patterns on neurologic outcome and survival at 6 months

\begin{tabular}{|c|c|c|c|c|c|c|}
\hline EEG pattern (day 0-5) & Survival, n (\%) & OR $(95 \% \mathrm{Cl})$ & $p$ Value & CPC 1-2, n (\%) & OR $(95 \% \mathrm{Cl})$ & $p$ Value \\
\hline Benign EEG pattern & $55 / 76(72.4)$ & 1.00 (referent) & & $54 / 76(71.0)$ & 1.00 (referent) & \\
\hline RSE pattern & $19 / 36(52.8)$ & $0.43(0.19-0.97)$ & 0.0431 & $16 / 36(44.4)$ & $0.33(0.13-0.80)$ & 0.0127 \\
\hline GPD pattern & $2 / 13(15.4)$ & $0.07(0.01-0.34)$ & 0.0010 & $0 / 13(0.0)$ & $0.02(0.00-0.12)$ & $<0.0001$ \\
\hline Malignant nonepileptiform EEG pattern & $1 / 41(2.4)$ & $0.01(0.00-0.07)$ & $<0.0001$ & $0 / 41(0.0)$ & $0.01(0.00-0.04)$ & $<0.0001$ \\
\hline Main effect of EEG patterns & & & 0.0001 & & & $<0.0001$ \\
\hline
\end{tabular}

Abbreviations: $\mathrm{Cl}=$ confidence interval; $\mathrm{CPC}=$ cerebral performance category; GPD = generalized periodic discharge; $\mathrm{OR}=$ odds ratio.

RSE was $4.7 \pm 4.3$ days after onset. Treatment was successful in terminating RSE in 35 (81.4\%) patients, while 8 (18.6\%) patients died during RSE.

Status epilepticus was refractory in all cases and superrefractory in $21(58.3 \%)$ cases, requiring treatment with anesthetics and multiple AEDs. The mean number of AEDs used was 3.2 (range 1-7). The mean number of anesthetic cycles was 2.4 (range 1-5), and the mean number of days of anesthesia was 4.8 (range 0.8-14.2). A total of 85 anesthetic cycles were used for treating RSE in the study population: a combination of propofol and midazolam was used in $72.3 \%$ of cycles, while thiopental was used in $21.2 \%$ and ketamine in $4.7 \%$.

\section{Long-term neurologic outcome and survival}

Considering the entire cohort, survival was achieved in 77 (46.4\%) patients and good neurologic outcome (CPC 1-2) in $70(42.2 \%)$ patients at 6 months (table 3$)$. The EEG pattern in the first 5 days after cardiac arrest was highly associated with both survival $(p=0.0001)$ and neurologic outcome $(p<0.0001)$.

Table 4 Effect of selected prognostic indicators on good neurologic outcome (top) and survival (bottom) in the study population

\begin{tabular}{|c|c|c|c|c|c|c|c|}
\hline & \multicolumn{2}{|c|}{ CPC 1-2 } & \multicolumn{2}{|c|}{ CPC 3-5 } & \multirow{2}{*}{ OR } & \multirow{2}{*}{$95 \% \mathrm{Cl}$} & \multirow{2}{*}{$p$ Value } \\
\hline & \multicolumn{2}{|c|}{ Median (IQR) or $\mathbf{n}(\%)$} & \multicolumn{2}{|c|}{ Median (IQR) or $\mathrm{n}(\%)$} & & & \\
\hline Low flow (min) for 1-min increase & 10 & $10-30$ & 40 & $22-61$ & 0.96 & $0.94-0.97$ & $<0.0001$ \\
\hline Absent pupillary reflexes & 0 & 0 & 24 & 100 & 0.02 & ne-0.11 & $<0.0001$ \\
\hline Absent corneal reflexes & 3 & 7 & 40 & 93 & 0.04 & $0.01-0.15$ & $<0.0001$ \\
\hline NSE for 1-unit increase & 24 & $24-49$ & 75 & $39-178$ & 0.98 & $0.96-0.99$ & 0.0006 \\
\hline Absent $\mathrm{N} 20$ responses & 1 & 5.3 & 19 & 94.7 & 0.03 & $0.00-0.19$ & $<0.0001$ \\
\hline Neuroimaging (severe anoxic brain injury) & 2 & 6.1 & 31 & 93.9 & 0.04 & $0.00-0.19$ & $<0.0001$ \\
\hline Absent EEG background reactivity & 5 & 6.7 & 75 & 93.8 & 0.03 & $0.01-0.08$ & $<0.0001$ \\
\hline
\end{tabular}

\begin{tabular}{|c|c|c|c|c|c|c|c|}
\hline \multirow{3}{*}{ Low flow (min) for 1-min increase } & \multirow{2}{*}{\multicolumn{2}{|c|}{$\frac{\text { Survivors }}{\text { Median (IQR) or } n(\%)}$}} & \multirow{2}{*}{\multicolumn{2}{|c|}{$\frac{\text { Nonsurvivors }}{\text { Median (IQR) or } n(\%)}$}} & \multirow{3}{*}{$\begin{array}{l}\text { OR } \\
0.96\end{array}$} & \multirow{3}{*}{$\begin{array}{l}95 \% \mathrm{Cl} \\
0.94-0.98\end{array}$} & \multirow{3}{*}{$\begin{array}{l}\boldsymbol{p} \text { Value } \\
<0.0001\end{array}$} \\
\hline & & & & & & & \\
\hline & 19 & $12-30$ & 40 & $21-61$ & & & \\
\hline Absent pupillary reflexes & 0 & 0 & 24 & 100 & 0.02 & $0.00-0.09$ & $<0.0001$ \\
\hline Absent corneal reflexes & 6 & 13.6 & 38 & 86.4 & 0.07 & $0.03-0.19$ & $<0.0001$ \\
\hline NSE for 1-unit increase & 38 & $24-54$ & 75 & $38-228$ & 0.98 & $0.97-0.99$ & 0.0007 \\
\hline Absent $\mathrm{N} 20$ responses & 3 & 15 & 17 & 85 & 0.08 & $0.02-0.28$ & $<0.0001$ \\
\hline Neuroimaging (severe anoxic brain injury) & 5 & 15.2 & 28 & 84.8 & 0.10 & $0.03-0.29$ & $<0.0001$ \\
\hline Absent EEG background reactivity & 11 & 13.8 & 69 & 86.2 & 0.06 & $0.03-0.29$ & $<0.0001$ \\
\hline
\end{tabular}

Abbreviations: $\mathrm{Cl}$ = confidence interval; $\mathrm{CPC}=$ cerebral performance category; IQR = interquartile range; NSE = neuron-specific enolase; $\mathrm{OR}=$ odds ratio. 
Patients with a benign EEG pattern had the highest chance of both survival (72.4\%) and good neurologic outcome (71.1\%) and were chosen as a reference to compare patients with other EEG patterns. Patients with an RSE pattern had a moderate reduction in survival (52.8\%, OR 0.43, 95\% CI 0.19-0.97) and good neurologic outcome (44.4\%, OR 0.33, 95\% CI 0.13-0.80). Patients with GPDs has a dramatic reduction in survival (15.4\%, OR 0.07, 95\% CI 0.01-0.34) and good neurologic outcome (0\%, OR 0.02, 95\% CI 0.00-0.12). An even more dramatic reduction was observed for patients with malignant nonepileptiform EEG pattern for both survival (2.4\%, OR 0.01, 95\% CI 0.00-0.07) and good neurologic outcome (0\%, OR 0.01, 95\% CI 0.00-0.04).

The effects of selected prognostic indicators (low-flow time, brainstem reflexes, N20, NSE, MRI, EEG background reactivity) on survival and neurologic outcome in our population are shown in table 4.

\section{Discussion}

The intensity and duration of treatment of postanoxic RSE are highly controversial issues. ${ }^{23,24}$ No evidence is currently available on whether a more aggressive treatment would substantially affect the final neurologic outcome in refractory postanoxic status epilepticus.

Aggressive and prolonged treatment carries the risk of futility, $^{25}$ while a low-intensity and short-lasting treatment carries the risk of self-fulfilling prophecies, i.e., negative outcome being the consequence of withdrawal of care due to (sometimes erroneous) prognostication of a negative outcome. ${ }^{26}$ This latter risk might be particularly high if prognostication relies on a single negative indicator such as refractory epileptiform EEG activity.

A multicenter randomized clinical trial (Treatment of Electroencephalographic Status Epilepticus After Cardiopulmonary Resuscitation [TELSTAR]) is ongoing to address this issue. ${ }^{27}$ In the TELSTAR trial, patients are being randomized to a stepwise, aggressive, EEG-guided treatment with AEDs and anesthetics for 48 hours vs standard care without EEG guidance.

In the present study, we reported survival and long-term neurologic outcome of a prospective cohort of post-cardiac arrest patients in coma for $>24$ hours who received a standardized aggressive treatment with AEDs and anesthetics if RSE was detected by cEEG monitoring. Intensity and duration of treatment were guided not only by EEG but also by multimodal prognostic indicators.

We adopted a strict definition of RSE, according to the International League Against Epilepsy definition, ACNS terminology, and Salzburg criteria. In particular, we made a clear distinction between low-frequency $(<2.5 \mathrm{~Hz})$ periodic discharges (GPDs) and RSE without periodic discharges, which were considered 2 mutually exclusive EEG patterns. In case of evolution from RSE to GPDs, patients were considered to have GPDs. Besides RSE and GPDs, we identified 2 other nonepileptiform EEG patterns based on background activity and reactivity that were called benign and malignant according to previous studies. ${ }^{28,29}$ These 4 mutually exclusive EEG patterns were easily and unequivocally identified in the first 5 days after cardiac arrest with cEEG monitoring and allowed a prognostic analysis.

Our results showed that patients with RSE represent a distinct population in term of indicators of severity of anoxic brain injury and may present a good neurologic prognosis in $>40 \%$ with a standardized, aggressive, and prolonged treatment. Our results are remarkably different from recent observational studies without aggressive standardized treatment, which reported $<10 \%$ of good neurologic outcome in postanoxic patients with RSE defined according to the ACNS terminology. ${ }^{30,31}$

Compared to patients with GPDs, patients with RSE displayed lower low-flow time and NSE levels and were less likely to show clinical motor seizures, absent N20 responses, and severe anoxic brain injury on neuroimaging. The 4 EEG patterns delineate a range of severity of anoxic brain injury and were strongly associated with long-term survival and neurologic prognosis, with patients with RSE appearing more similar to patients with a benign EEG pattern and patients with GPDs appearing more similar to patients with a malignant nonepileptiform EEG pattern.

We adopted a broad definition of clinical motor seizures, including any observable myoclonic, clonic, or tonic-clonic manifestation during the entire stay in the ICU. This definition has the advantage of taking into account the switching between convulsive and NCSE over time (and with therapy). Our findings indicate that the majority of RSE is nonconvulsive. Conversely, status myoclonus was far more common in GPDs compared to RSE and was associated with a negative outcome, as previously reported. ${ }^{1}$

Notably, no predefined limit of duration of treatment was applied. Onset of RSE was variable with a mean of 3 days after cardiac arrest, which could potentially be explained by the combined effect of hypothermia and sedation in the first 24 to 48 hours. In $>50 \%$ of cases, RSE lasted $>72$ hours, and in $\approx 30 \%$ of cases, RSE lasted $>7$ days. Aggressive treatment was continued as long as needed in case of super-RSE if multimodal prognostic indicators were not unfavorable. Therefore, treatment duration in our cohort is substantially longer than 48 hours, which is the predefined duration of treatment in the TELSTAR trial. Our findings suggest that prolonged treatment is necessary and beneficial in selected cases of postanoxic RSE and warn against early outcome prediction (and early decisions of withdrawal of care) in case of RSE.

Conversely, the GPD pattern was regarded as an unfavorable prognostic indicator on the basis of recent studies, which 
include GPDs within the highly malignant EEG patterns after cardiac arrest. ${ }^{29}$ Therefore, we adopted a less aggressive treatment in case of a GPD pattern, in most cases not exceeding 1 cycle of anesthetics-induced coma. Notably, no patient included in this study received withdrawal of care, intended as active reduction of respiratory support or vasoactive drugs. Although we could not definitely exclude the risk of self-fulfilling prophecies in patients with GPDs in our series, we think this is very unlikely in view of the current evidence.

The study has strengths and limitations. The first strength is the fairly large sample assessed and managed according to a standardized prognostic and therapeutic program. A second strength is the inclusion of patients from a single source, which helps minimize the variability of measures intrinsic in multicenter studies. A third strength is that no patient was lost to follow-up.

The first limitation is selection bias because this series is from a university hospital. Although we did our best to enroll consecutive patients, we cannot exclude different outcomes from patients enrolled in other centers and managed according to different prognostic and therapeutic programs. A second limitation is the use of hypothermia at $34^{\circ} \mathrm{C}$ for 24 hours for all patients included in this study, which may reduce generalizability to patients routinely treated with normothermia at $36^{\circ} \mathrm{C}$. Patients were recruited from 2011 to 2016 and were treated according to the American Heart Association guidelines (2010 and 2015), which recommend any target core temperature between $32^{\circ} \mathrm{C}$ and $36^{\circ} \mathrm{C}$, given the continuing debate on this topic. ${ }^{32} \mathrm{~A}$ third limitation is the use of a simplified EEG, instead of a full EEG, for continuous monitoring. However, 18-channel EEG was performed at least twice in the first 5 days to verify concordance with the cEEG. A fourth limitation is the uncontrolled setting, which prevented us from assessing the efficacy of our aggressive treatment schedule compared to a lower-intensity treatment schedule. Nonetheless, our main aim was to investigate the long-term prognosis of RSE compared to other EEG patterns in a cohort of postanoxic patients treated with a standardized aggressive treatment. We believe that our findings revealed some important issues in terms of patient selection and intensity and duration of treatment that could inspire the design of future randomized controlled trials comparing aggressive and conservative treatment of postanoxic status epilepticus.

\section{Author contributions}

S. Beretta: concept and design; acquisition, analysis, or interpretation of data; drafting of the manuscript; critical revision of the manuscript for important intellectual content. A. Coppo: concept and design; acquisition, analysis, or interpretation of data; critical revision of the manuscript for important intellectual content. E. Bianchi: statistical analysis; acquisition, analysis, or interpretation of data; critical revision of the manuscript for important intellectual content. C. Zanchi: acquisition, analysis, or interpretation of data; critical revision of the manuscript for important intellectual content. D. Carone, A. Stabile, G. Padovano, E. Sulmina, and A. Grassi: acquisition, analysis, or interpretation of data; critical revision of the manuscript for important intellectual content; administrative, technical, or material support. G. Bogliun: acquisition, analysis, or interpretation of data; critical revision of the manuscript for important intellectual content. G. Foti, C. Ferrarese, and A. Pesenti: acquisition, analysis, or interpretation of data; critical revision of the manuscript for important intellectual content; obtained funding. E. Beghi: concept and design; acquisition, analysis, or interpretation of data; drafting of the manuscript. L. Avalli: concept and design; acquisition, analysis, or interpretation of data; critical revision of the manuscript for important intellectual content.

\section{Acknowledgment}

The authors thank the medical and nursing staff of the ICU at San Gerardo Hospital ASST Monza for their support and collaboration and Dr. Francesco De Leo for his advice.

\section{Study funding}

This work was supported by the Italian Ministry of University and Research.

\section{Disclosure}

The authors report no disclosures relevant to the manuscript. Go to Neurology.org/ $\mathrm{N}$ for full disclosures.

\section{Publication history}

Received by Neurology January 20, 2018. Accepted in final form August 23, 2018.

\section{References}

1. Sandroni C, Cariou A, Cavallaro F, et al. Prognostication in comatose survivors of cardiac arrest: an advisory statement from the European Resuscitation Council and the European Society of Intensive Care Medicine. Resuscitation 2014;85:1779-1789.

2. Rittenberger JC, Popescu A, Brenner RP, Guyette FX, Callaway CW. Frequency and timing of nonconvulsive status epilepticus in comatose post-cardiac arrest subjects treated with hypothermia. Neurocrit Care 2012;16:114-122.

3. Mani R, Schmitt SE, Mazer M, Putt ME, Gaieski DF. The frequency and timing of epileptiform activity on continuous electroencephalogram in comatose post-cardiac arrest syndrome patients treated with therapeutic hypothermia. Resuscitation 2012; 83:840-847.

4. Youn CS, Callaway CW, Rittenberger JC, Service PCA. Combination of initial neurologic examination, quantitative brain imaging and electroencephalography to predict outcome after cardiac arrest. Resuscitation 2017;110:120-125.

5. Lybeck A, Friberg H, Aneman A, et al. Prognostic significance of clinical seizures after cardiac arrest and target temperature management. Resuscitation 2017;114: $146-151$.

6. Hofmeijer J, Tjepkema-Cloostermans MC, Blans MJ, Beishuizen A, van Putten MJ. Unstandardized treatment of electroencephalographic status epilepticus does not improve outcome of comatose patients after cardiac arrest. Front Neurol 2014;5:39.

7. Hirsch LJ, LaRoche SM, Gaspard N, et al. American Clinical Neurophysiology Society's standardized critical care EEG terminology: 2012 version. J Clin Neurophysiol 2013;30:1-27.

8. Beniczky S, Hirsch LJ, Kaplan PW, et al. Unified EEG terminology and criteria for nonconvulsive status epilepticus. Epilepsia 2013;54(suppl 6):28-29.

9. Leitinger M, Trinka E, Gardella E, et al. Diagnostic accuracy of the Salzburg EEG criteria for non-convulsive status epilepticus: a retrospective study. Lancet Neurol 2016; 15:1054-1062.

10. Ribeiro A, Singh R, Brunnhuber F. Clinical outcome of generalized periodic epileptiform discharges on first EEG in patients with hypoxic encephalopathy postcardiac arrest. Epilepsy Behav 2015;49:268-272.

11. Amorim E, Rittenberger JC, Zheng JJ, et al. Continuous EEG monitoring enhances multimodal outcome prediction in hypoxic-ischemic brain injury. Resuscitation 2016; 109:121-126.

12. Amorim E, Rittenberger JC, Baldwin ME, Callaway CW, Popescu A. Malignant EEG patterns in cardiac arrest patients treated with targeted temperature management who survive to hospital discharge. Resuscitation 2015;90:127-132. 
13. Rossetti AO, Oddo M, Liaudet L, Kaplan PW. Predictors of awakening from postanoxic status epilepticus after therapeutic hypothermia. Neurology 2009;72:744-749.

14. Matthews EA, Magid-Bernstein J, Presciutti A, et al. Categorization of survival and death after cardiac arrest. Resuscitation 2017;114:79-82.

15. Grossestreuer AV, Gaieski DF, Abella BS, et al. Factors associated with post-arrest withdrawal of life-sustaining therapy. Resuscitation 2017;110:114-119.

16. Stammet $\mathrm{P}$, Collignon $\mathrm{O}$, Hassager $\mathrm{C}$, et al. Neuron-specific enolase as a predictor of death or poor neurological outcome after out-of-hospital cardiac arrest and targeted temperature management at $33^{\circ} \mathrm{C}$ and $36^{\circ} \mathrm{C}$. J Am Coll Cardiol 2015;65:2104-2114.

17. Rossetti AO, Lowenstein DH. Management of refractory status epilepticus in adults: still more questions than answers. Lancet Neurol 2011;10:922-930.

18. Shorvon S, Ferlisi M. The treatment of super-refractory status epilepticus: a critical review of available therapies and a clinical treatment protocol. Brain 2011;134:2802-2818.

19. Trinka E, Cock H, Hesdorffer D, et al. A definition and classification of status epilepticus: report of the ILAE Task Force on Classification of Status Epilepticus. Epilepsia 2015;56:1515-1523.

20. Mlynash M, Campbell DM, Leproust EM, et al. Temporal and spatial profile of brain diffusion-weighted MRI after cardiac arrest. Stroke 2010;41:1665-1672.

21. Lee BK, Jeung KW, Song KH, et al. Prognostic values of gray matter to white matter ratios on early brain computed tomography in adult comatose patients after out-ofhospital cardiac arrest of cardiac etiology. Resuscitation 2015;96:46-52.

22. Hsu CH, Li J, Cinousis MJ, et al. Cerebral performance category at hospital discharge predicts long-term survival of cardiac arrest survivors receiving targeted temperature management. Crit Care Med 2014;42:2575-2581.
23. Cronberg T. Should postanoxic status epilepticus be treated agressively? Yes! J Clin Neurophysiol 2015;32:449-451.

24. Dragancea I, Wise MP, Al-Subaie N, et al. Protocol-driven neurological prognostication and withdrawal of life-sustaining therapy after cardiac arrest and targeted temperature management. Resuscitation 2017;117:50-57.

25. Rossetti AO. Should postanoxic status epilepticus be treated aggressively?-No! J Clin Neurophysiol 2015;32:447-448.

26. Geocadin RG, Peberdy MA, Lazar RM. Poor survival after cardiac arrest resuscitation: a self-fulfilling prophecy or biologic destiny?. Crit Care Med 2012;40: 979-980.

27. Ruijter BJ, van Putten MJ, Horn J, et al. Treatment of Electroencephalographic Status Epilepticus After Cardiopulmonary Resuscitation (TELSTAR): study protocol for a randomized controlled trial. Trials 2014;15:433.

28. Rossetti AO, Rabinstein AA, Oddo M. Neurological prognostication of outcome in patients in coma after cardiac arrest. Lancet Neurol 2016;15:597-609.

29. Westhall E, Rossetti AO, van Rootselaar AF, et al. Standardized EEG interpretation accurately predicts prognosis after cardiac arrest. Neurology 2016;86:1482-1490.

30. Dragancea I, Backman S, Westhall E, Rundgren M, Friberg H, Cronberg T. Outcome following postanoxic status epilepticus in patients with targeted temperature management after cardiac arrest. Epilepsy Behav 2015;49:173-177.

31. Backman S, Westhall E, Dragancea I, et al. Electroencephalographic characteristics of status epilepticus after cardiac arrest. Clin Neurophysiol 2017;128:681-688.

32. Polderman $\mathrm{KH}$, Varon J. Confusion around therapeutic temperature management hypothermia after in-hospital cardiac arrest? Circulation 2018;137:219-221. 


\section{Neurology}

\section{Neurologic outcome of postanoxic refractory status epilepticus after aggressive treatment}

Simone Beretta, Anna Coppo, Elisa Bianchi, et al.

Neurology published online October 31, 2018

DOI 10.1212/WNL.0000000000006615

This information is current as of October 31, 2018

\section{Updated Information \&} Services

Subspecialty Collections

Permissions \& Licensing

\section{Reprints}

including high resolution figures, can be found at: http://n.neurology.org/content/early/2018/10/31/WNL.0000000000006 615.full

This article, along with others on similar topics, appears in the following collection(s):

Antiepileptic drugs

http://n.neurology.org/cgi/collection/antiepileptic_drugs

Coma

http://n.neurology.org/cgi/collection/coma

EEG

http://n.neurology.org/cgi/collection/eeg_

Prognosis

http://n.neurology.org/cgi/collection/prognosis

Status epilepticus

http://n.neurology.org/cgi/collection/status_epilepticus

Information about reproducing this article in parts (figures,tables) or in its entirety can be found online at:

http://www.neurology.org/about/about_the_journal\#permissions

Information about ordering reprints can be found online:

http://n.neurology.org/subscribers/advertise

Neurology ${ }^{\circledR}$ is the official journal of the American Academy of Neurology. Published continuously since 1951, it is now a weekly with 48 issues per year. Copyright @ 2018 American Academy of Neurology. All rights reserved. Print ISSN: 0028-3878. Online ISSN: 1526-632X.

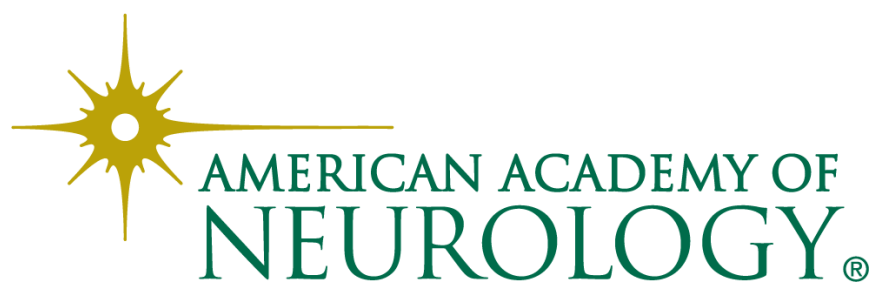

